\title{
AtCTF7 is required for establishment of sister chromatid cohesion and association of cohesin with chromatin during meiosis in Arabidopsis
}

\author{
Dipesh K Singh, Sebastien Andreuzza ${ }^{\dagger}$, Aneesh P Panoli ${ }^{\dagger}$ and Imran Siddiqi ${ }^{*}$
}

\begin{abstract}
Background: The establishment of sister chromatid cohesion followed by its controlled release at the metaphase to anaphase transition is necessary for faithful segregation of chromosomes in mitosis and meiosis. Cohesion is established by the action of Ctf7/Eco1 on the cohesin complex during DNA replication following loading of cohesin onto chromatin by the Scc2-Scc4 complex. Ctf7 is also required for sister chromatid cohesion during repair of DNA double strand breaks. Ctf7 contains an acetyltransferase domain and a zinc finger motif and acetylates conserved lysine residues in the Smc3 subunit of cohesin. In Arabidopsis CTF7 is encoded by a single gene and mutations in AtCTF7 cause embryo lethality indicating that the gene is essential.

Results: To study the function of Ctf7 in plants and to determine its role in sister chromatid cohesion, we constructed a conditional allele of AtCTF7 in Arabidopsis using an inducible RNA interference (RNAi) strategy, so as to avoid the embryo lethality caused by mutations in AtCTF7. We found that induction of RNAi against AtCTF7 caused severe inhibition and defects in growth during vegetative and reproductive stages as well as sterility. AtCTF7-RNAi plants displayed chromosome fragmentation and loss of sister chromatid cohesion during meiosis. Immunostaining for the cohesion subunit AtSCC3 showed a marked reduction in association of cohesin with chromatin during meiosis in AtCTF7-RNAi plants.
\end{abstract}

Conclusions: We find that AtCTF7 is essential for sister chromatid cohesion during meiosis in Arabidopsis and is required for association of cohesin with chromatin in prophase of meiosis.

Keywords: Plant meiosis, Chromosome organization, Synapsis, Conditional RNAi, Gametogenesis, DNA repair

\section{Background}

Proper chromosome segregation during cell division requires that sister chromatids produced by DNA replication are held together until their controlled separation at anaphase. This function is accomplished by the cohesin complex, whose conserved core subunits consist of the Structural Maintenance of Chromosome (SMC) proteins Smc1 and Smc3, the Sister Chromatid Cohesion (SCC) protein Scc3, and the $\alpha$-kleisin protein Scc1 [1]. According to the ring model of cohesin action, Smc1 and Smc3 interact to form a $\mathrm{V}$ shaped heterodimer, closed by Scc1 with the help of Scc3, to form a ring that is considered to entrap sister chromatids and hold them

\footnotetext{
* Correspondence: imran@ccmb.res.in

${ }^{\dagger}$ Equal contributors

Centre for Cellular \& Molecular Biology (CSIR), Uppal Road, Hyderabad 500007, India
}

physically together $[2,3]$. Cohesion is released at anaphase by the cleavage of Scc1 by separase, a protease that is activated by the anaphase promoting complex/ cyclosome (APC/C) [3].

In Saccharomyces cerevisiae, cohesion is established by $\mathrm{Ctf} 7 / \mathrm{Eco1}$, after cohesin has been loaded on chromatin by the Scc2-Scc4 complex [4-6]. Ctf7 establishes cohesion during $\mathrm{S}$ phase, and interacts with components of the DNA replication machinery, including PCNA and RFC [5-7]. These results led to a model in which sister chromatid cohesion is established concomitantly with DNA replication [8]. Ctf7 encodes a zinc finger protein with an active acetyltransferase domain, and it was found that Ctf7 acetylation of Smc3 on conserved lysines, was critical for establishment of cohesion by counteracting the Wpl1-Pds5 complex in preventing establishment of cohesion [9-14]. Establishment of

\section{Biomed Central}


cohesion has been suggested to occur in concert with lagging strand synthesis [15], and Smc3 acetylation leading to establishment of functional cohesion occurs only in association with replication [16]. Recycling of the Smc3 subunit is aided by deacetylation by Hos1 following cleavage of Scc1 by separase to release cohesion at the metaphase to anaphase transition, and is important for establishment of cohesion [17-19]. The eso1-H17 mutant in Schizosaccharomyces pombe exhibits delayed mitosis as a result of activation of the spindle checkpoint, and defective segregation of chromosomes in mitosis [20]. In Drosophila, mutations in Deco result in altered distribution of cohesin at metaphase, and premature entry into anaphase [21]. In humans, mutations in ESCO2 cause Roberts syndrome which results from a deficiency of cohesion around the centromeres, and encompasses a number of developmental abnormalities as well as mental retardation and renal and cardiac dysfunction [22].

The machinery for establishment of cohesion is conserved in Arabidopsis, and homologues of Scc2 and Ctf7 have been identified and functionally characterized. Mutations in AtSCC2 and AtCTF7 result in embryo lethality, however AtCTF7 is dispensable for endosperm growth [23,24]. Interestingly, AtCTF7 was found to possess acetyltransferase activity in vitro, and could complement the yeast ctf7-203 mutant, suggesting conserved biochemical function with its yeast counterpart [23]. By using a conditional RNA interference (RNAi) approach, it has been demonstrated that $A t S C C 2$ is required during meiosis for sister chromatid cohesion, chromosomal axis formation and synapsis between homologues [24]. The function of AtCTF7 in establishment of sister chromatid cohesion in planta remains to be shown.

Here, we used a conditional RNAi approach to examine the role of AtCTF7 in sister chromatid cohesion, and to analyze the effects of the loss of AtCTF7 during vegetative and reproductive development. We found that downregulation of AtCTF7 severely inhibited growth during vegetative and reproductive stages, and resulted in both male and female sterility. During meiosis, AtCTF7-RNAi lines displayed typical loss of cohesion phenotypes, including abnormal chromosome organization, impaired chromosome synapsis and DNA fragmentation. Consistent with an expected involvement of AtCTF7 in cohesion, we found that sister chromatid cohesion was lost at both chromosome arms and centromeres in AtCTF7-RNAi plants. Finally, we found that AtSCC3 localization on chromatin was compromised during meiosis in AtCTF7-RNAi plants, indicating that AtCTF7 is required for association of cohesin with chromosomes in Arabidopsis, a feature that appears to be similar to Drosophila, where Deco is required for Scc1 association with chromosomes during $M$ phase [21]. Overall, our results establish an essential role for
AtCTF7 in vegetative development and in sister chromatid cohesion during meiosis.

\section{Results}

\section{AtCTF7 is required for growth during vegetative and reproductive stages}

To examine the requirement for AtCTF7 during different stages of development we constructed a conditional allele using an inducible RNAi approach to deplete AtCTF7 mRNA. A hairpin RNAi construct for AtCTF7 was made and placed under control of the dexamethasoneinducible transactivator LhGR [25]. T1 transformants were grown and seeds collected. T2 plants were then grown and treated with dexamethasone either at the vegetative stage or after bolting. Treatment during the vegetative stage resulted in defective growth in both aerial and root tissues, accompanied by loss of greening (Figure $1 \mathrm{~A}$ and $\mathrm{B}$, Additional file 1: Figure S1), indicating that AtCTF7 is required for plant growth during vegetative stages. Treatment with dexamethasone following bolting resulted in strong inhibition of growth of the inflorescence as well as sterility (Figure 1C). Pollen was largely sterile and inviable following dexamethasone treatment (Figure 1D-G), and lacked clearly defined sperm and vegetative nuclei (Figure $1 \mathrm{~L}$ and $\mathrm{M}$, Additional file 1: Figure S2). Ovules in treated plants showed arrest in female gametogenesis starting at an early stage (1n) as well as missing embryo sacs (Figure 1H-K). A proportion of ovules also showed defects in integument development (Figure 1J). A total of 29 independent transformants were examined, out of which 5 lines showed strong growth defects and sterility following treatment with dexamethasone. Quantitative comparison of AtCTF7 expression indicated a reduction in treated compared to untreated control plants (Additional file 1: Figure S3), consistent with the growth defects being due to depletion of AtCTF7. Examination of AtCTF7 promoter activity using a $P_{A t C T F 7}$ nlsGUS reporter indicated that the AtCTF7 promoter is active in both the shoot and root meristematic regions (Figure $2 \mathrm{~A}$ and $2 \mathrm{C}$ ), as well as in young buds and leaves (Figure 2A and 2B). Expression is reduced in older leaves and flowers (Figure 2B). Within reproductive cells, expression was observed in pollen and in the female gametophyte (Figure 2D and 2E), and is consistent with the defects in gametogenesis described above for AtCTF7-RNAi plants.

These results indicate that AtCTF7 is expressed in dividing cells and is required for normal development and growth during both vegetative and reproductive stages.

\section{Knockdown of AtCTF7 results in defects in sister chromatid cohesion and chromosome organization during meiosis}

To examine whether AtCTF7 is required in meiosis, chromosome spreads were carried out on male meiocytes 

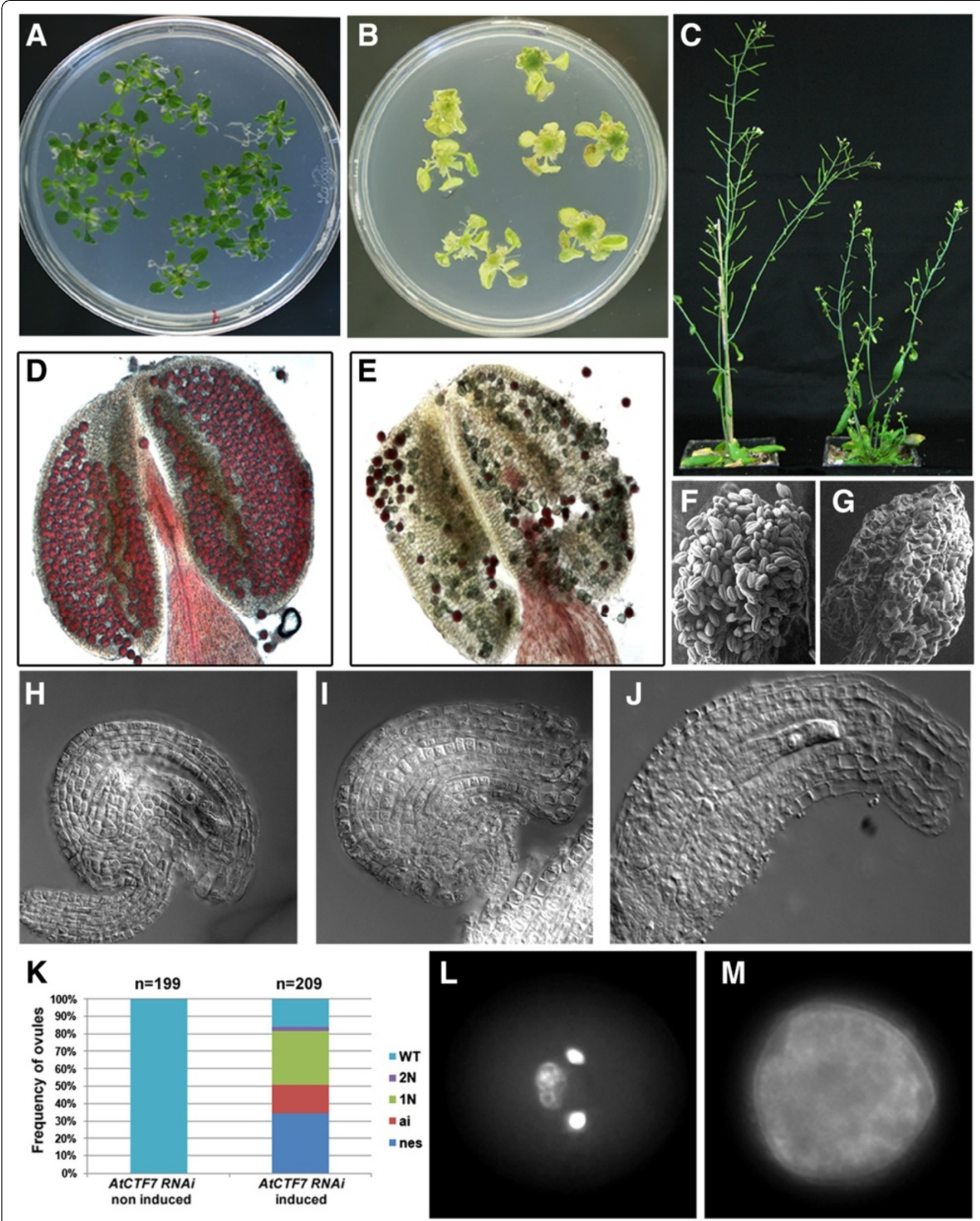

Figure 1 (See legend on next page.) 
(See figure on previous page.)

Figure 1 Knockdown of AtCTF7results in growth defects and sterility. The RNAi mediated down-regulation of AtCTF7 results in growth inhibition, female and male gametophyte defects, and sterility. (A,B) Loss of coloration and growth inhibition in treated (B) compared to untreated (A) AtCTF7-RNAi seedlings. (C) Impaired growth and sterile siliques in treated (right) compared to untreated (left) AtCTF7-RNAi adult plants. (D,E) Alexander staining of mature anthers shows loss of pollen viability (green grains instead of purple) in treated (E) compared to untreated (D) AtCTF7-RNAi mature anthers. (F,G) Scanning electron micrograph of anther from untreated (F) and treated (G) AtCTF7-RNAi plant showing reduction of pollen grains following treatment. (H-J) Impaired female gametophyte and ovule development in treated $(\mathbf{I}, \mathbf{J})$ compared to untreated (H) AtCTF7-RNAi plants. (I) Ovule lacking a female gametophyte. (J) Ovule with abnormal integument development. (K) Quantification of female gametophytic and ovule defects shown in (I) and (J) for ovule stages 3-4 to 3-6 [26]. Gametophytes were classified as uninucleate $(1 \mathrm{~N})$, binucleate $(2 \mathrm{~N})$, missing (nes), or wild type (WT) if they contained greater than 2 nuclei. ai: ovules showing abnormal integument development. (L,M) DAPI staining of mature pollen grains reveals loss of chromatin in the male gametophyte of treated (M) compared to untreated (L) AtCTF7-RNAi plants, which contains two sperm cells and one vegetative cell.

from AtCTF7-RNAi plants (Figure 3). Plants that had not been treated with dexamethasone showed normal pairing and synapsis, and normal organization of meiotic chromosomes (Figure 3A-H). Early prophase stages were marked by the appearance of thin chromosome threads at leptotene, followed by zygotene and pachytene stages showing normal pairing and synapsis of chromosomes (Figure 3A-D). Subsequent late prophase stages and meiosis I and II stages, were also seen to occur normally in untreated plants (Figure 3E-H). In contrast, plants that were treated with dexamethasone showed severe defects in meiotic chromosome organization (Figure 3I-L). The chromosomal defects were apparent early in prophase I for which the characteristic stages could not be clearly distinguished. Chromosomes appeared disorganized and had a patchy appearance

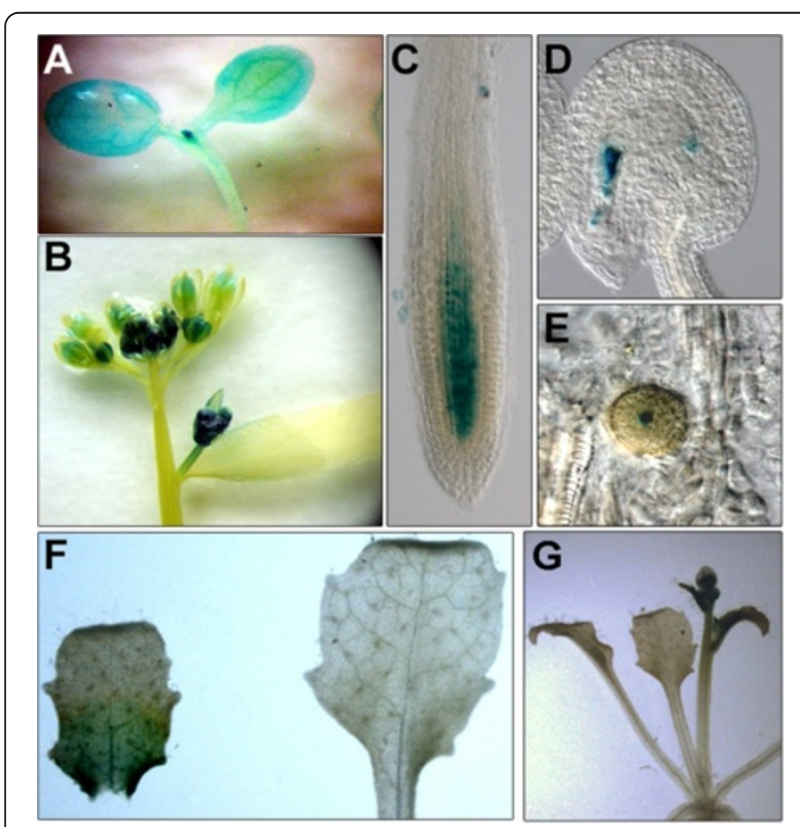

Figure 2 AtCTF7 is expressed in actively dividing tissues. Examination of a $P_{\text {AtCTF }}$ nls GUS reporter shows expression in dividing tissues. (A) Shoot meristematic region and cotyledonary leaves. (B) Inflorescence and young buds. (C) Root meristem. (D) Female gametophyte. (E) Pollen. (F) Young cauline leaf (left) and expanded rosette leaf (right). (G) Portion of whole plant showing GUS expression in inflorescence but not in rosette leaves. with discontinuities. The synizetic knot did not form and mid to late prophase stages were marked by chromosomes appearing clumped and highly fragmented (Figure 3I-K). Late prophase stages were characterized by the presence of condensed fragments of chromosomes which could exceed 20 in number indicating that fragmentation had taken place (Figure 3L).

To examine the role of AtCTF7 in sister chromatid cohesion and pairing at the centromeric region, we carried out fluorescence in situ hybridization (FISH) using a centromere repeat probe that hybridizes to all the centromeres [24]. In untreated control plants, 8-10 centromere signals (mean $=9.5 ; \mathrm{n}=33$ ) could be clearly detected at early prophase stages (leptotene) in meiocytes, whereas at zygotene and pachytene stages, 3-5 signals (mean $=3.1 ; \mathrm{n}=72$ ) were observed (Figure $4 \mathrm{~A}-\mathrm{L}$ ). For dexamethasone treated plants, the number of signals at early prophase ranged between 9 and 20 (mean $=12.0$; $\mathrm{n}=64$ ). The presence of greater than 10 signals at prophase I indicated loss of centromere cohesion in AtCTF7-RNAi plants (Figure 4M-X). In addition there was variability in shape of the signal in AtCTF7-RNAi plants compared to untreated control plants which suggested defects in centromere organization. These results indicate that AtCTF7 is required for pairing, cohesion, and proper organization of centromeric regions. To examine arm cohesion we used a BAC probe specific for chromosome 4 (Figure 5). In untreated plants, we observed two signals in early prophase, and a single signal in meiocytes at mid-prophase stages when chromosomes had fully synapsed (Figure 5A-F), whereas in plants that were treated with dexamethasone, we observed more than two signals (Figure 5G-L). Overall the results indicate that AtCTF7 is required for both centromere and arm cohesion.

\section{Reduced association of cohesin with chromatin in AtCTF7-RNAi plants}

In order to examine the role of AtCTF7 in sister chromatid cohesion and chromosome organization,we examined the localization of the cohesin subunit AtSCC3 [27], and the axial element protein ASY1 [28] during 

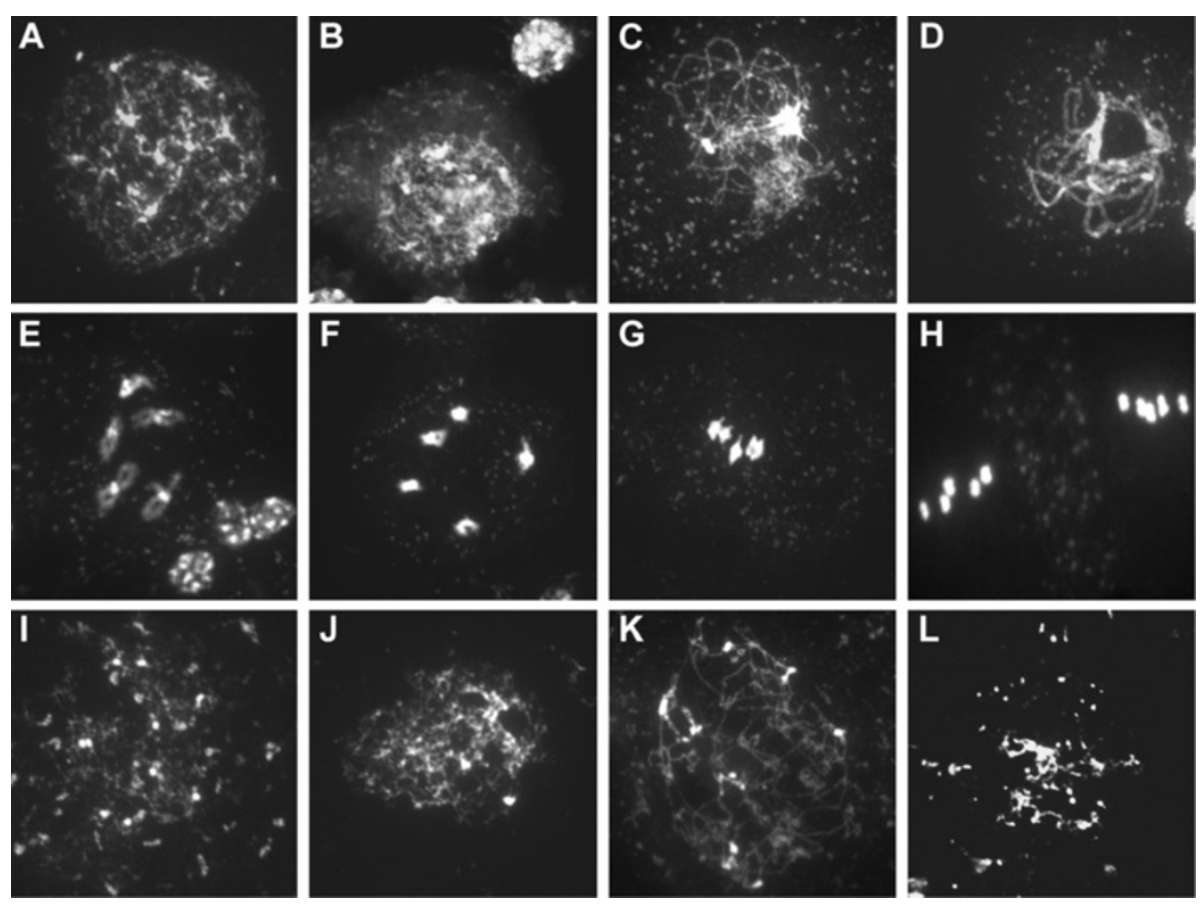

Figure 3 Defective meiotic chromosome organization in AtCTF7-RNAi. Acid spreads of male meiotic chromosomes stained with DAPI. (A-H) Untreated AtCTF7-RNAi line, (I-L) AtCTF7-RNAi line treated with dexamethasone. (A) Meiotic interphase. (B) Leptotene. (C) Zygotene. (D) Pachytene showing complete chromosome synapsis. (E) Late diplotene showing 5 partially condensed bivalents. (F) Diakinesis showing 5 condensed bivalents. (G) Metaphase I showing 5 aligned bivalents. (H) Metaphase II showing two sets of 5 aligned chromosomes. (I) Early prophase stage showing few thread-like segments and many discontinuities. (J) Early prophase stage with discontinuous and fragmented chromatin. (K) Mid-prophase showing unpaired, discontinuous chromatin and absence of synizetic knot. (L) Late prophase stage, showing condensed and fragmented chromatin.

male meiosis (Figure 6, Additional file 1: Figure S4). In untreated control plants, AtSSC3 immunostaining extended throughout the chromatin at early prophase stages, and marked chromosomal axes during zygotene and pachytene stages (Figure 6A-P). In contrast, the level of AtSCC3 immunostaining, was greatly reduced in male meiocytes from AtCTF7-RNAi plants from early prophase onwards (Figure 6Q-AF). ASY1 immunostaining in control plants is seen at early prophase and marks the chromosome axes at leptotene (Figure 6C, G, K, and $\mathrm{O})$. The staining pattern overlaps with that of AtSCC3 (Figure 6D, H, L, P). In the case of AtCTF7-RNAi plants, association of ASY1 with chromatin was comparable to that of control plants (Figure 6S, W, AA, AE). Thus, although ASY1 staining of chromatin shows significant overlap with that of AtSCC3, its association with chromatin appears to be independent of AtSCC3 which is known to precede ASY1, and is also consistent with analysis of the Atscc3-1 mutant [27].

\section{Discussion}

Ctf7/Eco1 proteins have been shown to control establishment of cohesion in yeast, Drosophila, and mammals $[5,6,20,21,29]$. In the case of plants, the Arabidopsis homolog of Ctf7 (AtCTF7) has been shown to be required for embryo development but not required for development of the endosperm [23], leaving open the possibility of a Ctf7-independent mechanism for sister chromatid segregation operating in meiosis. Evidence for a Ctf7-independent mechanism for sister chromatid segregation in yeast is based on the viability of an eco1 $\Delta$ wpl1 $\Delta$ strain [30]. A dosage dependent role for $\mathrm{Ctf} 7$ in meiosis in yeast has been suggested based on haploinsufficiency during sporulation [15]. Establishment of sister chromatid cohesion in meiosis in yeast may therefore be more sensitive to reduced dosage of $\mathrm{Ctf} 7$ than in mitosis. Alternatively, acetylation of other proteins by Ctf7 during meiosis may also be involved [31]. A role for $\mathrm{Ctf} 7$ in meiosis is also suggested from an examination of the localization and regulation of murine ESCO2 [32], however a requirement for $\mathrm{Ctf} 7$ in sister chromatid cohesion specifically during meiosis remains to be established. In this study we have shown using Arabidopsis as a model, that AtCTF7 is also required for sister chromatid cohesion in meiosis.

The conditional RNAi approach to examine the function of AtCTF7 in plants revealed defects in sister chromatid cohesion in meiosis. The establishment of sister 

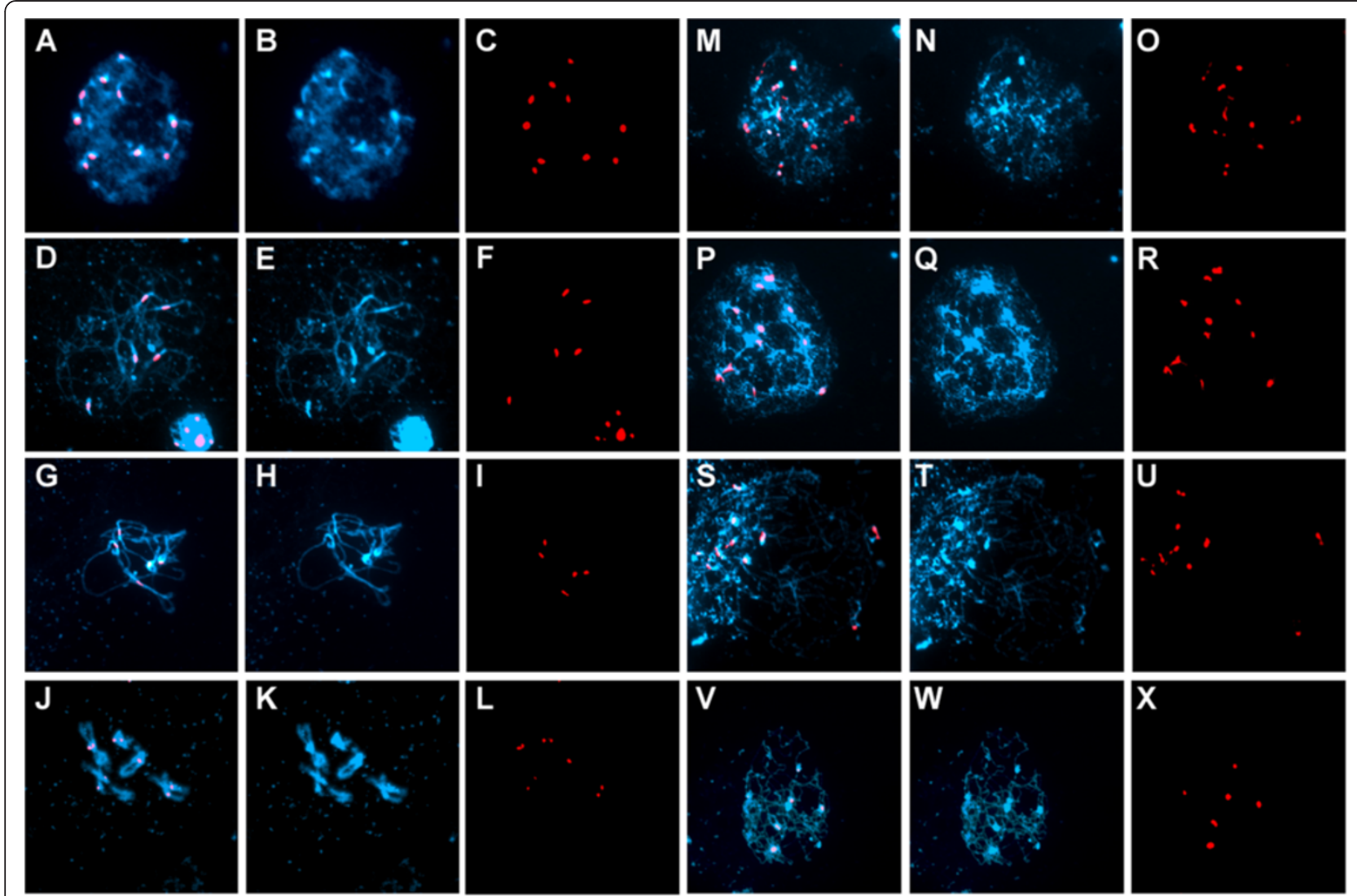

Figure 4 Loss of centromeric pairing and cohesion in AtCTF7-RNAi male meiocytes. FISH of male meiotic chromosome spreads hybridized with a centromeric probe showing DAPI (blue) and probe (red). (A-L) Untreated transgenic AtCTF7-RNAi line, (M-X) transgenic AtCTF7-RNAi line treated with dexamethasone. For each panel, left column shows merged images of DAPI and the probe, the middle column shows DAPI, and the right column shows the probe alone. (A-C) Early leptotene cell showing 10 centromeres. (D-F) Late zygotene showing 5 centromeric signals as a result of synapsis of centromeres. (G-I) Pachytene showing 5 centromeric signals as synapsis of chromosomes is complete. (J-L) Diplotene. (M-O, P-R) Early meiotic prophase, showing more than 10 centromeric signals. (S-U, V-X) Mid meiotic prophase stages, showing more than 10

centromeric signals $\mathbf{( S - U )}$ and 6 centromeric signals $(\mathbf{V}-\mathbf{X})$.

chromatid cohesion in both arm and centromeric regions during meiosis was dependent upon AtCTF7. The meiotic phenotypes comprised defects early in prophase which presented as discontinuities in the thread-like appearance characteristic of leptotene and zygotene stages. In the most severe cases, the thread-like structure was largely absent and the chromatin appeared highly fragmented. At later prophase stages, the fragmented phenotype was further apparent by the presence of a large number of separated and condensed chromatin fragments. The results are consistent with a failure (in meiosis) to repair double strand breaks for which Ctf7 is known to be required [33]. The fragmentation phenotype is similar to that observed for Atrec 8 and Atmnd1 mutants, which are defective in repair of meiotic double strand breaks [34,35]. However, since the RNAi strategy employed is not specific to meiosis, the possibility that the fragmentation phenotype may also be influenced by depletion of AtCTF7 earlier during mitosis in the progenitor cells of the meiocytes is not ruled out.
Arabidopsis mutants defective in both cohesion and formation of meiotic double strand breaks do not display such fragmentation phenotypes $[27,36]$.

We observed severe defects in vegetative as well as reproductive growth and development, pointing to a role for AtCTF7 throughout the plant life cycle, and extending previous work showing an essential requirement for AtCTF7 in embryo development [23]. A $P_{\text {AtCTF }}$ nlsGUS reporter was strongly expressed in root and shoot meristems, and in young buds and leaves. In young developing leaves, a polarity in expression was observed with GUS staining confined to the basal part of the leaf and absent towards the distal portion. The gradient of expression is similar to that for the cell division marker CycB1;1::GUS [37]. Expression declined in older buds and was not observed in expanded rosette leaves. The expression of AtCTF7 is thus seen to occur in tissues that are undergoing active cell division, consistent with the known involvement of $\mathrm{Ctf} 7$ in promoting establishment of cohesion in conjunction with DNA 


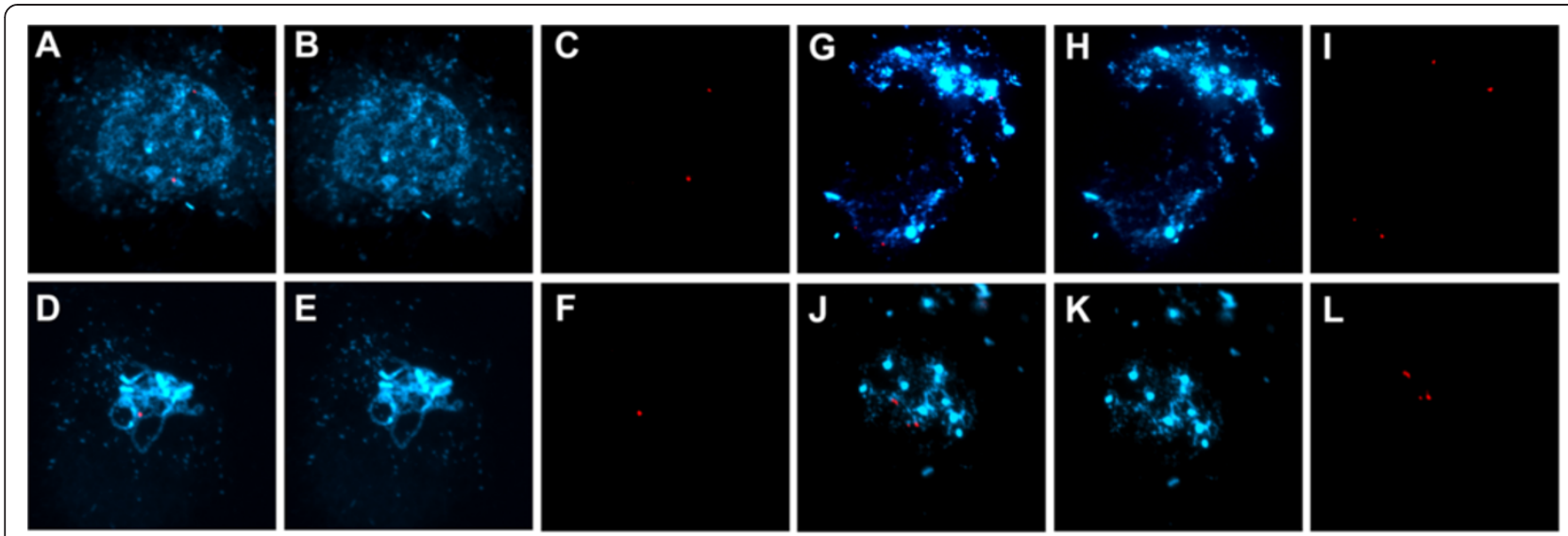

Figure 5 Loss of chromosome arm cohesion and impaired synapsis during male meiosis in AtCTF7-RNAi. FISH of male meiotic

chromosome spreads hybridized with a chromosome 4 arm probe showing chromosomes stained with DAPI (blue) and probe (red). (A-F) Untreated transgenic AtCTF7-RNAi line; (G-L) transgenic AtCTF7-RNAi line treated with dexamethasone. For each set, the left image shows DAPI and the probe merged images, the middle image shows DAPI, and the right image shows the probe alone. (A-C) Early leptotene showing 2 signals. (D-F) Pachytene showing a single probe signal due to complete synapsis of chromosomes. (G-I) Early prophase showing 4 signals, indicating loss of chromatid cohesion. (J-L) Mid-prophase stage showing 3 signals.

replication [8]. Within reproductive cells, expression was observed in pollen and in the female gametophyte. The sterile phenotype we observed is likely to be accounted for mainly by the defects in meiosis as well as a possible contribution from a gametophytic component.

Establishment of cohesion by $\mathrm{Ctf} 7$ involves acetylation of conserved lysine residues in the Smc3 subunit of cohesion which inhibits the action of the Wpl1-Pds5 complex in preventing establishment of cohesion [9,10,12-14]. In yeast, Drosophila, and human cells, $\mathrm{Ctf} 7 / \mathrm{Eco} 1$ is required for the establishment of cohesion but not for association of cohesin with chromatin in interphase [5,21,29]. In Drosophila, a deco mutant shows reduced staining for the cohesin subunit Scc1 at prometaphase of mitosis [21]. The strong reduction in association of AtSCC3 with chromatin in early meiotic

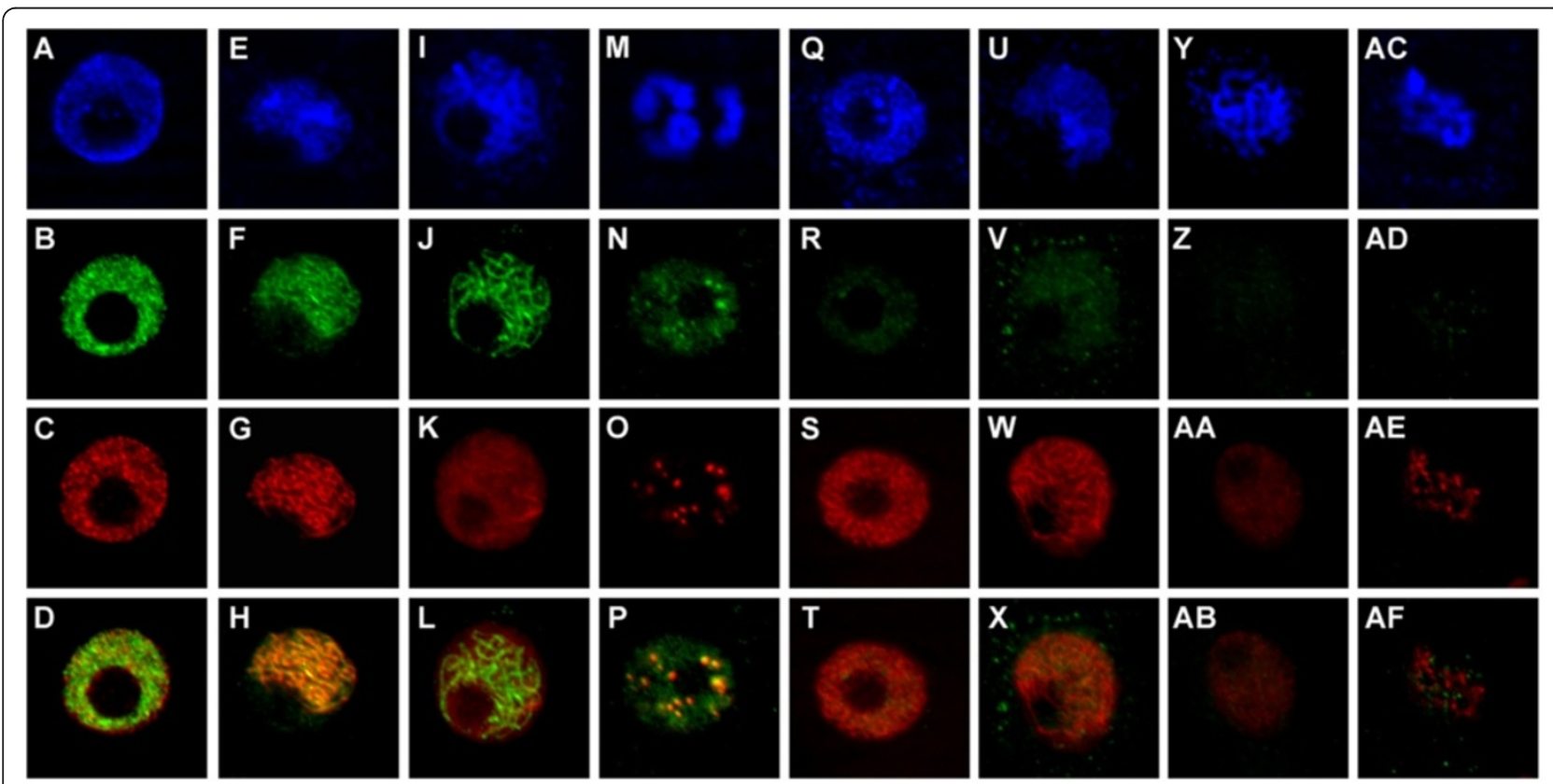

Figure 6 Impaired loading of AtSCC3 cohesin on meiotic chromosomes in AtCTF7-RNAi. Immunostaining of anther squashes showing chromosomes stained with DAPI (blue), AtSCC3 (green), ASY1 (red) and merged images for the green and red channels (bottom row). (A-P), untreated transgenic AtCTF7-RNAi line, (Q-AF) transgenic AtCTF7-RNAi line treated with dexamethasone. (A-D, Q-T) Early leptotene. (E-H, U-X) Late leptotene. (I-L, Y-AB) Pachytene. (M-P, AC-AF) Diplotene. 
prophase as revealed by immunostaining of meiocytes in AtCTF7-RNAi plants is similar to what has been observed for the deco mutant in prometaphase of mitosis, and suggests conservation of Ctf7/Ecol function in plants.

\section{Conclusions}

In conclusion, our findings show that AtCTF7 is required for establishment of sister chromatid cohesion during meiosis in Arabidopsis, and that continued association of cohesin with chromatin in meiosis depends on AtCTF7.

\section{Methods}

\section{Plant materials and growth conditions}

The Arabidopsis thaliana strains used were of the Columbia ecotype (Col-0). Plants were grown as described in [38]. To generate transgenic Arabidopsis, constructs were mobilized into Agrobacterium tumefaciens strain AGL-1 using triparental mating, and transformed into Arabidopsis by vacuum infiltration as described in [39]. $P_{A t C T F 7} n l s G U S$ was transformed into wild-type Col, and AtCTF7-RNAi was transformed into a line carrying a $P_{C a M V 35 S} L h G R-N$ transgene [25]. Transgenic plants were selected on MS media, containing $120 \mu \mathrm{g} / \mathrm{ml}$ gentamycin (Sigma-Aldrich) for AtCTF7RNAi transformants, and $50 \mu \mathrm{g} / \mathrm{ml}$ kanamycin for $P_{A t C T F 7 n} n l s G U S$ transformants, and were further confirmed by PCR.

\section{Cloning procedures}

The AtCTF7 promoter, comprising 646 bp upstream of the ATG and $45 \mathrm{bp}$ from AtCTF7 coding sequence, was amplified by PCR using primers Fctf7gusHindIII and Rctf7gusnlsBamH1, and cloned as a BamH1-HindIII fragment in frame with a nlsGUS tag in the pBI101.2 binary vector. For the RNAi construct, 658 bp fragments were amplified by PCR using primer pairs F1rnaiXba1 and R1rnaiBamH1, and F1rnaiXho1 and R1rnaiEcoR1, and cloned as Xba1-BamH1 and Xho1-EcoR1 fragments in opposite directions in pKANNiBAL [40]. The RNAi cassette was excised as a XhoI-BamH1 fragment and cloned into the binary vector pZP222-6xPOP described in [25].

\section{Dexamethasone treatment}

Transgenic seeds were germinated on MS plates, and grown for 7 days after which they were transferred on MS plate containing $20 \mu \mathrm{M}$ dexamethasone (Sigma). Seedlings were analyzed for phenotypes 7 days after transfer on dexamethasone plates. Treatment of adult plants after bolting was carried out by inclusion of $20 \mu \mathrm{M}$ dexamethasone in the watering solution which was delivered by subirrigation. Samples for meiotic analysis were collected for analysis 5 days after the start of dexamethasone treatment.

\section{RNA isolation and quantitative RT-PCR}

Total RNA was isolated using Trizol (Invitrogen) following the manufacturer's protocol. cDNA synthesis was performed using Reverse Transcription System (Invitrogen SuperScript II) and oligo(dT) primers. Real Time PCR reactions were performed using SYBR Green PCR master mix (Applied Biosystems). GAPC was used as the internal normalization control. PCR was performed on the ABI Prism 7900 HT Sequence Detection System (Applied Biosystems) in a 384 well reaction plate according to the manufacturer's recommendations. Primers used were Ctf7qRTF and Ctf7qRTR for AtCTF7, and GAPRTF and GAPRTR for GAPC (Additional file 1: Table S1). Cycling parameters consisted of 2 minutes incubation at $50^{\circ} \mathrm{C}, 10$ minutes at $95^{\circ} \mathrm{C}$, and 40 cycles of $95^{\circ} \mathrm{C}$ for 15 seconds, $57^{\circ} \mathrm{C}$ for $30 \mathrm{sec}-$ onds and $67^{\circ} \mathrm{C}$ for 30 seconds. The PCR reaction was performed in triplicate for each RNA sample, and the experiment was carried out on two different biological samples representing the same RNAi line. Specificity of the amplifications was verified at the end of each PCR run using $A B I$ prism dissociation curve analysis software. Results from the ABI Prism 7900 HT Sequence Detection System were analyzed further using Microsoft Excel. Relative amounts of mRNA were calculated from threshold points (Ct values) located in the log-linear range of real time PCR amplification plots using the 2- $\Delta \mathrm{Ct}$ method. Standard deviations in Additional file 1: Figure S1 are for variation across biological samples.

\section{Cytological procedures}

Whole mount analysis of ovules was done after fixing and clearing the inflorescence in methyl benzoate as described previously [38]. Scanning electron microscopic (SEM) analysis of pollen was carried out using a Hitachi scanning electron microscope (model $3400 \mathrm{~N}$, http://www.hitachi-hitec.com). Pollen viability was examined using Alexander staining [41]. For DAPI analysis of pollen, anthers were squashed and stained with DAPI $(1 \mathrm{\mu g} / \mathrm{ml})$. Meiotic chromosome spreads were carried out as described in [42], with minor modifications [43]. Observations were made on a Zeiss Axioplan 2 imaging microscope, using a Plan Apochromat $63 \times$ oil immersion objective. Tissue from $P_{A t C T F}$ nls GUS transgenic plants was stained for GUS activity as described in [38].

For FISH, chromosome spreads were carried out as described above, and FISH analysis was carried out according to the method described in [44], with minor modifications [35]. The 180-bp centromeric pAL1 repeat was used to detect centromere sequences [45]. A plasmid harboring two copies of the pAL1 repeat was 
subjected to PCR in the presence of Cy3-dATP (GE Healthcare), using PAL forward and reverse primers (Additional file 1: Table S1). BAC clones T19F6 and T22A6 from chromosome 4 were used as probes to monitor arm cohesion after being subjected to nick translation and labeling by Cy3-dATP (Roche). Slides were observed under a Zeiss Axioplan 2 imaging microscope equipped with a Plan Apochromat $63 \times$ oil immersion objective, using an excitation (Cy3, $550 \mathrm{~nm})$ and long-pass emission (Cy3, $570 \mathrm{~nm}$ ) filter.

For immunostaining, inflorescences were fixed as described in [46]. Young buds were dissected out and washed with $10 \mathrm{mM}$ Citrate Buffer pH4.5 (1× CB), followed by digestion with a cell wall digesting enzyme mix containing $0.3 \%$ cellulase, $0.3 \%$ pectolyase, $0.4 \%$ cytohelicase (all Sigma) in $1 \times \mathrm{CB}$, and incubated for $30 \mathrm{~min}$ at $37^{\circ} \mathrm{C}$. The enzyme mix was replaced with $1 \times$ PBS, and anthers were dissected out from buds on a slide and squashed using a $22 \times 22 \mathrm{~mm}$ coverslip. The slide was snap-frozen by dipping in liquid nitrogen and the coverslip was immediately removed. Slides were then dried and dipped briefly in molten $1 \%$ gelatin, $1 \%$ agarose solution to cover the cells with a thin layer of gelatin-agarose and dried. Slides were rehydrated in $1 \times$ PBS, and digested with the enzyme mix described above for $30 \mathrm{~min}$ at $37^{\circ} \mathrm{C}$. This was followed by permeabilization of the cells in $1 \times$ PBS, $1 \%$ Triton-X100, for $30 \mathrm{~min}$ and washing of the slides $2-3$ times in $1 \times$ PBS containing $0.1 \%$ Triton-X100. Immunostaining was performed as described in [24], using ASY1 antibody at a 1:1000 dilution, and AtSCC3 antibody at 1:200 dilution. All secondary antibodies were used at a dilution of $1: 100$. Slides were mounted in $1 \mathrm{ug} / \mathrm{ml}$ DAPI in Vectashield (VectorLabs). Cells were imaged using a Zeiss Axio Imager.Z1 microscope equipped with an apotome module, using a Plan-Apochromat $63 \times$ oilimmersion objective.

While this manuscript was under review, a related study appeared online by Bolanos-Villegas et al., on the role of AtCTF7 in DNA repair, mitosis, and meiosis [47].

\section{Additional file}

Additional file 1: Figure S1. Effect of dexamethasone treatment on wild type. Figure S2. Microscopic analysis of pollen in AtCTF7-RNAi. Figure S3. Quantitative reverse transcription PCR (q-RT-PCR) of AtCTF7 in AtCTF7-RNAi. Figure S4. Raw pictures of AtSCC3 and ASY1

immunostaining on untreated and treated AtCTF7-RNAi line. Table S1. List of primers used.

\section{Competing interests}

The authors declare that they have no competing interests in the work presented in this study.

\section{Authors' contributions}

IS, DS, and AP designed the experiments. DS performed the experiments with contributions from SA. DS, IS, and SA prepared the manuscript. All authors read and approved the final manuscript.

\section{Acknowledgements}

We thank Christopher Franklin and Raphael Mercier for gifts of antibodies. DS thanks S. Prabha for assistance. This work was supported by funds from the Council of Scientific and Industrial Research (CSIR), Govt. of India, and by a Centre of Excellence Grant from the Department of Biotechnology to IS. DS was supported by a fellowship from the Indian Council of Medical Research (ICMR), and SA was supported by a CSIR International Fellowship.

Received: 8 April 2013 Accepted: 5 August 2013

Published: 14 August 2013

\section{References}

1. Nasmyth $\mathrm{K}$, Haering $\mathrm{CH}$ : Cohesin: Its roles and mechanisms. Annu Rev Genet 2009, 43:525-558.

2. Rudra S, Skibbens RV: Cohesin codes - interpreting chromatin architecture and the many facets of cohesin function. J Cell Sci 2013, 126:31-41.

3. Nasmyth $\mathrm{K}$ : Cohesin: a catenase with separate entry and exit gates? Nat Cell Biol 2011, 13:1170-1177.

4. Ciosk R, Shirayama M, Shevchenko A, Tanaka T, Toth A, Shevchenko A, Nasmyth K: Cohesin's binding to chromosomes depends on a separate complex consisting of scc2 and scc4 proteins. Mol Cell 2000, 5:243-254.

5. Tóth A, Ciosk R, Uhlmann F, Galova M, Schleiffer A, Nasmyth K: Yeast cohesin complex requires a conserved protein, $\mathrm{Eco} 1 \mathrm{p}(\mathrm{Ctf7})$, to establish cohesion between sister chromatids during DNA replication. Genes Dev 1999, 13:320-333.

6. Skibbens RV, Corson LB, Koshland D, Hieter P: Ctf7p is essential for sister chromatid cohesion and links mitotic chromosome structure to the DNA replication machinery. Genes Dev 1999, 13:307-319.

7. Moldovan G-L, Pfander B, Jentsch S: PCNA controls establishment of sister chromatid cohesion during S phase. Mol Cell 2006, 23:723-732.

8. Sherwood R, Takahashi TS, Jallepalli PV: Sister acts: coordinating DNA replication and cohesion establishment. Genes Dev 2010, 24:2723-2731

9. Sutani T, Kawaguchi T, Kanno R, Itoh T, Shirahige K: Budding yeast wpl1 (rad61)-pds5 complex counteracts sister chromatid cohesionestablishing reaction. Curr Biol 2009, 19:492-497.

10. Ben-Shahar TR, Heeger S, Lehane C, East P, Flynn H, Skehel M, Uhlmann F: Eco1-dependent cohesin acetylation during establishment of sister chromatid cohesion. Science 2008, 321:563-566.

11. Heidinger-Pauli JM, Ünal E, Koshland D: Distinct targets of the eco1 acetyltransferase modulate cohesion in S phase and in response to DNA damage. Mol Cell 2009, 34:311-321.

12. Rowland BD, Roig MB, Nishino T, Kurze A, Uluocak P, Mishra A, Beckouët $F$, Underwood P, Metson J, Imre R, Mechtler K, Katis VL, Nasmyth K: Building sister chromatid cohesion: Smc3 acetylation counteracts an antiestablishment activity. Mol Cell 2009, 33:763-774.

13. Ünal E, Heidinger-Pauli JM, Kim W, Guacci V, Onn I, Gygi SP, Koshland DE: A molecular determinant for the establishment of sister chromatid cohesion. Science 2008, 321:566-569.

14. Zhang J, Shi X, Li Y, Kim B-J, Jia J, Huang Z, Yang T, Fu X, Jung SY, Wang Y, Zhang P, Kim S-T, Pan X, Qin J: Acetylation of Smc3 by Eco1 is required for $\mathrm{S}$ phase sister chromatid cohesion in both human and yeast. Mol. Cell 2008, 31:143-151.

15. Rudra S, Skibbens RV: Sister chromatid cohesion establishment occurs in concert with lagging strand synthesis. Cell Cycle 2012, 11:2114-2121.

16. Song J, Lafont A, Chen J, Wu FM, Shirahige K, Rankin S: Cohesin acetylation promotes sister chromatid cohesion only in association with the replication machinery. J Biol Chem 2012, 287:34325-34336.

17. Rivera T, Losada A: Recycling cohesin rings by deacetylation. Mol Cell 2010, 39:657-659.

18. Beckouët $F$, Hu B, Roig MB, Sutani $T$, Komata M, Uluocak P, Katis VL, Shirahige K, Nasmyth K: An Smc3 acetylation cycle is essential for establishment of sister chromatid cohesion. Mol Cell 2010, 39:689-699.

19. Borges V, Lehane C, Lopez-Serra L, Flynn H, Skehel M, Ben-Shahar TR, Uhlmann F: Hos 1 deacetylates Smc3 to close the cohesin acetylation cycle. Mol Cell 2010, 39:677-688. 
20. Tanaka K, Yonekawa T, Kawasaki Y, Kai M, Furuya K, Iwasaki M, Murakami H, Yanagida M, Okayama $\mathrm{H}$ : Fission yeast eso1p is required for establishing sister chromatid cohesion during S phase. Mol Cell Biol 2000, 20:3459-3469.

21. Williams BC, Garrett-Engele CM, Li Z, Williams EV, Rosenman ED, Goldberg ML: Two putative acetyltransferases, San and Deco, are required for establishing sister chromatid cohesion in Drosophila. Curr Biol 2003, 13:2025-2036.

22. Vega H, Waisfisz Q, Gordillo M, Sakai N, Yanagihara I, Yamada M, van Gosliga D, Kayserili H, Xu C, Ozono K, Jabs EW, Inui K, Joenje H: Roberts syndrome is caused by mutations in Esco2, a human homolog of yeast Eco1 that is essential for the establishment of sister chromatid cohesion. Nat Genet 2005, 37:468-470.

23. Jiang L, Yuan L, Xia M, Makaroff CA: Proper levels of the Arabidopsis cohesion establishment factor Ctf7 are essential for embryo and megagametophyte, but not endosperm, Development. Plant Physiol 2010, 154:820-832.

24. Sebastian J, Ravi M, Andreuzza S, Panoli AP, Marimuthu MPA, Siddiqi I: The plant adherin AtSCC2 is required for embryogenesis and sisterchromatid cohesion during meiosis in Arabidopsis. Plant J 2009, 59:1-13.

25. Craft J, Samalova M, Baroux C, Townley H, Martinez A, Jepson I, Tsiantis M, Moore I: New pOp/LhG4 vectors for stringent glucocorticoid-dependent transgene expression in Arabidopsis. Plant J 2005, 41:899-918.

26. Schneitz K, Hülskamp M, Pruitt RE: Wild-type ovule development in Arabidopsis thaliana: a light microscope study of cleared whole-mount tissue. Plant J 1995, 7:731-749.

27. Chelysheva L, Diallo S, Vezon D, Gendrot G, Vrielynck N, Belcram K, Rocques N, Márquez-Lema A, Bhatt AM, Horlow C, Mercier R, Mézard C, Grelon M: AtREC8 and AtSCC3 are essential to the monopolar orientation of the kinetochores during meiosis. J Cell Sci 2005, 118:4621-4632.

28. Armstrong SJ, Caryl AP, Jones GH, Franklin FCH: Asy1, a protein required for meiotic chromosome synapsis, localizes to axis-associated chromatin in Arabidopsis and Brassica. J Cell Sci 2002, 115:3645-3655.

29. Hou F, Zou H: Two human orthologues of Eco1/Ctf7 acetyltransferases are both required for proper sister-chromatid cohesion. Mol Biol Cell 2005, 16:3908-3918.

30. Guacci V, Koshland D: Cohesin-independent segregation of sister chromatids in budding yeast. Mol Biol Cell 2012, 23:729-739.

31. Ghosh S, Gardner JM, Smoyer CJ, Friederichs JM, Unruh JR, Slaughter BD, Alexander R, Chisholm RD, Lee KK, Workman JL, Jaspersen SL: Acetylation of the SUN protein Mps3 by Eco1 regulates its function in nuclear organization. Mol Biol Cell 2012, 23:2546-2559.

32. Evans EB, Hogarth C, Evanoff RM, Mitchell D, Small C, Griswold MD: Localization and regulation of murine $\mathrm{EscO} 2$ during male and female meiosis. Biol Reprod 2012, 87:61.

33. Lu S, Goering M, Gard S, Xiong B, McNairn AJ, Jaspersen SL, Gerton JL: Eco1 is important for DNA damage repair in S cerevisiae. Cell Cycle 2010, 9:3315-3327.

34. Bai X, Peirson BN, Dong F, Xue C, Makaroff CA: Isolation and characterization of SYN1, a RAD21-like gene essential for meiosis in Arabidopsis. Plant Cell 1999, 11:417-430.

35. Panoli AP, Ravi M, Sebastian J, Nishal B, Reddy TV, Marimuthu MP, Subbiah $\checkmark$, Vijaybhaskar $V$, Siddiai I: AtMND1 is required for homologous pairing during meiosis in Arabidopsis. BMC Mol Biol 2006, 7:24.

36. Mercier R, Armstrong SJ, Horlow C, Jackson NP, Makaroff CA, Vezon D, Pelletier G, Jones GH, Franklin FCH: The meiotic protein SWI1 is required for axial element formation and recombination initiation in Arabidopsis. Development 2003, 130:3309-3318.

37. Donnelly PM, Bonetta D, Tsukaya H, Dengler RE, Dengler NG: Cell cycling and cell enlargement in developing leaves of Arabidopsis. Dev Biol 1999 215:407-419.

38. Siddiqi I, Ganesh G, Grossniklaus U, Subbiah V: The dyad gene is required for progression through female meiosis in Arabidopsis. Development 2000, 127:197-207.

39. Bechtold N, Pelletier G: In planta Agrobacterium-mediated transformation of adult Arabidopsis thaliana plants by vacuum infiltration. Methods $\mathrm{Mol}$ Biol 1998, 82:259-266.

40. Wesley SV, Helliwell CA, Smith NA, Wang M, Rouse DT, Liu Q, Gooding PS, Singh SP, Abbott D, Stoutjesdijk PA, Robinson SP, Gleave AP, Green AG, Waterhouse PM: Construct design for efficient, effective and highthroughput gene silencing in plants. Plant J 2001, 27:581-590.
41. Alexander MP: Differential staining of aborted and nonaborted pollen. Stain Technol 1969, 44:117-122.

42. Ross KJ, Fransz P, Jones GH: A light microscopic atlas of meiosis in Arabidopsis thaliana. Chromosome Res 1996, 4:507-516.

43. Agashe B, Prasad CK, Siddiqi I: Identification and analysis of DYAD: a gene required for meiotic chromosome organisation and female meiotic progression in Arabidopsis. Development 2002, 129:3935-3943.

44. Fransz P, Armstrong S, Alonso-Blanco C, Fischer TC, Torres-Ruiz RA, Jones G: Cytogenetics for the model system Arabidopsis thaliana. Plant J 1998, 13:867-876.

45. Martinez-Zapater JM, Estelle MA, Somerville CR: A highly repeated DNA sequence in Arabidopsis thaliana. Mol Gen Genet 1986, 204:417-423.

46. Cai X, Dong F, Edelmann RE, Makaroff CA: The Arabidopsis SYN1 cohesin protein is required for sister chromatid arm cohesion and homologous chromosome pairing. J Cell Sci 2003, 116:2999-3007.

47. Bolaños-Villegas $P$, Yang $X$, Wang HJ, Juan CT, Chuang MH, Makaroff CA, Jauh GY: Arabidopsis Chromosome Transmission Fidelity 7 (AtCTF7/ ECO1) is required for DNA repair, mitosis and meiosis. Plant J 2013. doi:10.1111/tpj.12261.

doi:10.1186/1471-2229-13-117

Cite this article as: Singh et al:: AtCTF7 is required for establishment of sister chromatid cohesion and association of cohesin with chromatin during meiosis in Arabidopsis. BMC Plant Biology 2013 13:117.

\section{Submit your next manuscript to BioMed Central and take full advantage of:}

- Convenient online submission

- Thorough peer review

- No space constraints or color figure charges

- Immediate publication on acceptance

- Inclusion in PubMed, CAS, Scopus and Google Scholar

- Research which is freely available for redistribution 